\title{
Forecast China's monthly urban fixed asset investment based on the ARIMA model
}

\author{
Jianglei Yuan ${ }^{1 *}$, Yaning $\mathrm{Li}^{2}$ and Yayu $\mathrm{Li}^{3}$ \\ ${ }^{1}$ School of Economics and Management, Beijing Jiaotong University, Beijing, 100000, China \\ ${ }^{2}$ School of Law and Public Administration, Hebei Normal University, Shijiazhuang City, Hebei Province, 050000, China \\ ${ }^{3}$ Quzhou County Shangshu Middle School, Handan City, Hebei Province, 057250, China
}

\begin{abstract}
In recent years, as China's urbanization level has risen, China's urban fixed asset investment has also been rising. Judging from monthly data, China's urban fixed asset investment has shown a volatile upward trend, with an obvious 11-month cycle. And in each cycle, the fluctuation range of the investment amount is getting larger. This paper uses an ARIMA model with an additive seasonal effect to fit the monthly urban fixed asset investment sequence and predict the future investment. In the end, this paper established a fitting model for China's urban fixed asset investment, and obtained a good forecasting effect.
\end{abstract}

\section{Introduction}

As the level of China's economic development continues to rise, the level of urbanization in China is also constantly being provided. The increase in the level of urbanization includes not only the increase in the urban housing population, but also the increase in the area of urban housing and the expansion of infrastructure. In the process of accelerating the pace of urbanization, China's annual investment in urban fixed assets is also increasing. Investment in fixed assets mainly includes investment in road construction and park expansion. As shown in Figure 1 , in recent years, China's urban fixed asset investment level has continued to increase as a whole, from 1301.403 billion yuan in February 2010 to 49792 billion yuan in July 2019. This total of 104 months of investment data is a typical time series data. Moreover, this process of improvement shows the characteristics of rising volatility, showing the characteristics of cyclical fluctuations with an 11 -month cycle. This is because the data obtained in this article contains only 11 months per year, and the investment in urbanization construction will change with the changes in the months of the year. For example, in 2016, the highest investment amount was 7,068,898 million yuan in June, and the lowest was $3,800.78$ billion yuan in February. The goal of this article is to predict the future data (August 2019-July 2020) on the basis of these existing data (February 2010-July 2019).

The ARIMA model is a model specially used to predict this kind of time series data. It mainly captures the characteristics of past historical data, such as growth trends and volatility characteristics, to predict the future data of the model. As shown in Figure 1, the annual fluctuation range of the investment amount is becoming wider and wider, showing that the fluctuation range has a strong increasing trend, so this article uses the ARIMA model with additive seasonal effect to fit the model.

\section{Sample data processing}

\subsection{Draw a line chart}

This paper selects China's monthly urban fixed asset investment (100 million yuan) data as sample data, and selects the time interval from February 2010 to July 2019. As shown in Figure 1, the line chart shows that the monthly urban fixed asset investment increases month by month in the form of an approximate linear function curve, and has a seasonal effect with an 11-month cycle. The intra-season volatility increases with the increase in investment, but this variation does not show a drastic change. Therefore, this paper sets the model as an additive seasonal model.

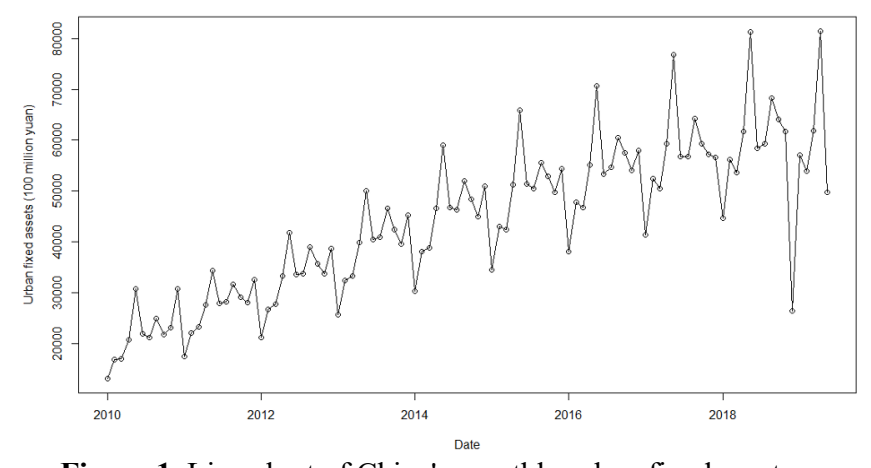

Figure 1. Line chart of China's monthly urban fixed asset investment 


\subsection{Sequence factor decomposition}

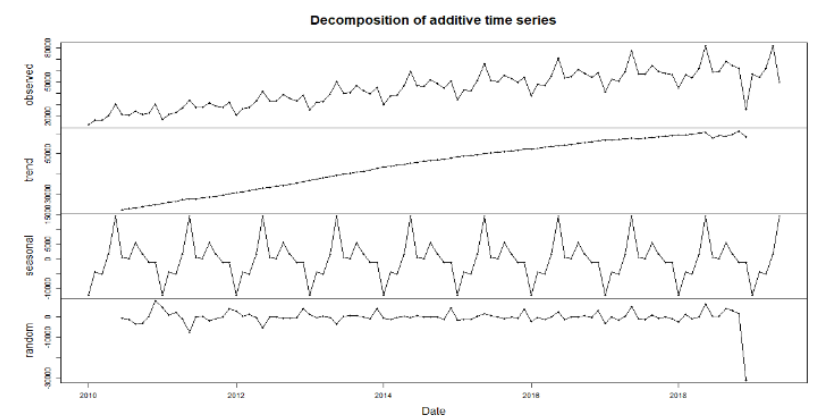

Figure 2. China's monthly urban fixed asset investment sequence factor breakdown diagram

As shown in Figure 2, this article decomposes China's monthly urban fixed asset investment into three parts: trend factors, seasonal factors, and random factors. It can be seen from the trend factors that China's monthly urban fixed asset investment has an obvious linear long-term increasing trend; from the seasonal factors, it can be seen that the time series has obvious seasonal factors with an 11-month cycle, so an 11-step difference process will be considered for this time series.

\subsection{Stationarity test: ADF test}

Considering that China's monthly urban fixed asset investment has an obvious linear growth trend, this paper makes a first-order difference on the original series, and then makes a lagging 11-period first-order difference on the difference series. After extracting the trend effect and seasonal effect of the original sequence, the sequence after the difference is similarly stable. The ADF stationarity test is performed on the sequence after the difference. At the $5 \%$ confidence level, the sequence is stable after the difference.

\subsection{Model ordering}

Draw the autocorrelation graph and partial autocorrelation graph of the sequence after the difference, as shown in Figure 3. The autocorrelation graph shows the autocorrelation coefficient 1 st order and 4th order truncation, the partial autocorrelation graph shows 2nd order truncation or 3rd order truncation, so try to use $\operatorname{ARMA}((1,4),(1,11), 2), \operatorname{ARMA}((1,4),(1,11), 3)$ extracts the short-term autocorrelation information of the sequence after the difference.

\section{3 design model}
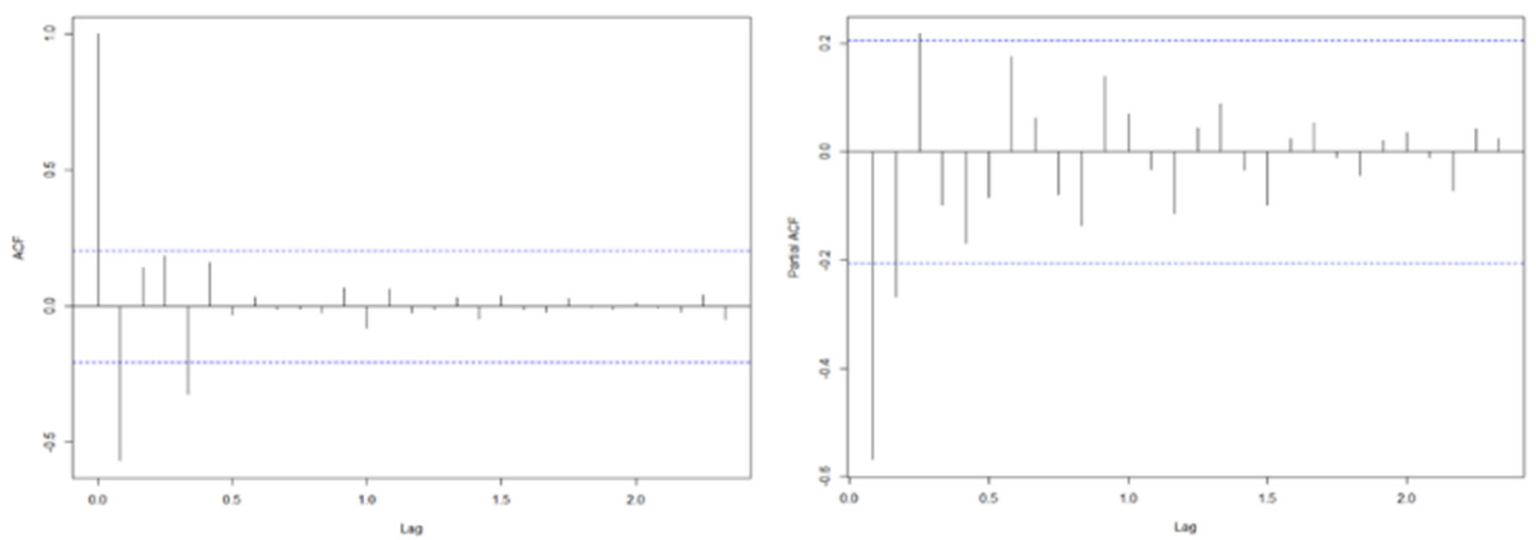

Figure 3. Sequence autocorrelation graph and partial autocorrelation graph

Based on the above information, a total of two addition models are established, as shown in Table 2.

\section{4 predictions and conclusions}

The model fitting is generally more accurate. Regardless of the time series stabilization in the early stage or the

white noise test of the model residuals in the later stage, the model passed perfectly, so the final prediction results given by the model are convincing and credible. At the same time, it must be noted that there are some areas for improvement in this model fitting: sharp fluctuations at the end may reduce the confidence of the prediction results.

As shown in Figure 4, there are sharp and sudden violent fluctuations at the end of the sequence after

Table 1. Model fitting expression.

\begin{tabular}{ccc}
\hline Model type & model & Model expression \\
\hline \multirow{2}{*}{ Additive model } & $\mathrm{M} 1$ & $(1-\mathrm{B})\left(1-\mathrm{B}^{11}\right) \mathrm{x}_{\mathrm{t}}=\frac{\left(1+1.2147 \mathrm{~B}-0.3643 \mathrm{~B}^{4}\right) \varepsilon_{\mathrm{t}}}{\left(1+0.4854 \mathrm{~B}-0.0099 \mathrm{~B}^{2}\right)}$ \\
\cline { 2 - 3 } & $\mathrm{M} 2$ & $(1-\mathrm{B})\left(1-\mathrm{B}^{11}\right) \mathrm{x}_{\mathrm{t}}=\frac{\left(1+1.2356 \mathrm{~B}-0.3731 \mathrm{~B}^{4}\right) \varepsilon_{\mathrm{t}}}{\left(1+0.4896 \mathrm{~B}-0.1074 \mathrm{~B}^{2}-0.3991 \mathrm{~B}^{3}\right)}$ \\
\hline
\end{tabular}

the difference, so the predictions made also fluctuate greatly with the violent fluctuations at the end of the data. But from the overall view of the sequence, the predicted volatility is obviously too large to be suspicious. The reason why the model paper does not show the significance of each coefficient of the model is because the 
model coefficients fitted in this paper are less significant. Although the quality of the overall model is relatively good, the deficiencies in this part also bring some flaws to the overall model construction.

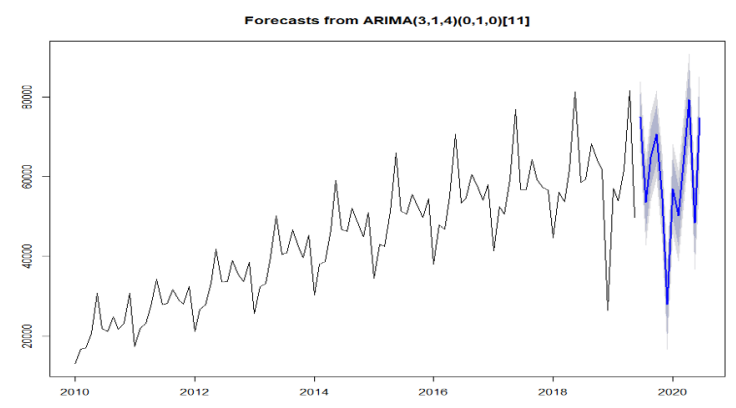

Figure 4. Predicted value map of Model 2

\section{References:}

1. Zou Shenghua."Research on GDP Forecast of Ji'an City Based on ARIMA Model." Open Journal of Social Sciences 08.12(2020): doi:10.4236/JSS.2020.812029.

2. Yan Jingjing."Evaluating the healthcare practice by defining healthcare principles: An autoregressive integrated moving average model based on time series." The International journal of health planning and management.(2020):. doi:10.1002/HPM.3101.

3. Yan Jingjing."Evaluating the healthcare practice by defining healthcare principles: An autoregressive integrated moving average model based on time series." The International Journal of Health Planning and Management 36.2(2020): doi:10.1002/HPM.3101.

4. Md. Salauddin Khan,and Umama Khan."Comparison of Forecasting Performance with VAR vs. ARIMA Models Using Economic Variables of Bangladesh." Asian Journal of Probability and Statistics .(2020):.

doi:10.9734/AJPAS/2020/V10I230243. 